\title{
A comparative analysis of 3-D representations of urban flood map in virtual environments for hazard communication purposes
}

\author{
Rosa De Santis ${ }^{1}$, Francesco Macchione ${ }^{1}$, Pierfranco Costabile ${ }^{1}$, and Carmelina Costanzo ${ }^{1}$ \\ ${ }^{1}$ LAMPIT (Laboratorio di Modellistica numerica per la Protezione Idraulica del Territorio), \\ Environmental and Chemical Engineering, University of Calabria, 87036 Rende, Italy.
}

\begin{abstract}
The flood hazard/risk maps do not allow a non-expert audience an immediate perception of the flooding impacts. Therefore, we need to modernize maps providing new communication approaches. In this context, 3-D representations of flood inundation through emerging formats in virtual and augmented realities may be considered as a powerful tool to engage users with flood hazards.

The challenge of the research is to create a virtual 3-D environment aimed at supporting the public, practitioners and decision-makers in interpreting and understanding the impact of simulated flood hazards. For this purpose, the paper aims to perform a comparative analysis of two techniques to carry out the 3-D realistic visualizations of a flood map for representing a potential flooding of the Crati River, in the old town of Cosenza (South of Italy). The first approach develops a simple and quick workflow that provides an overall look at a neighbourhood level, but reveals some limits in water level visualization at the individual buildings scale. The second one requires additional terrestrial laser scanning (TLS) acquisition and overcomes some limits of the first approach, by providing a visual insight about water level close to building façades.
\end{abstract}

\section{Introduction}

The activities associated with flood risk communication are related to the public perception through strategies linked to effective computational data and tools.

The flood hazard and risk maps are the traditional tools for risk management. They represent the flood-prone areas outlined by chromatic shading or contour based resulting from hydraulic numerical modelling. A flood hazard map, because unavoidably generalizes the information conveyed to the mapping scale reducing the detail [1], does not allow a nonexpert audience an immediate readability of flooding effects. The state of art about the flood mapping comprehension reveals that this tool often does not provide a strong support to stakeholders in flood risk management [2]. Since the importance of practitioners participation has been highlighted $[3,4]$, the development of new risk visual tools should simplify the identification of flood hazard and suggest feasible approaches to enhance flood hazard perception, to raise awareness in all stakeholders and influence behavior of people in emergency. 
An effective tool might be represented by visualizations of flood inundation in a 3-D virtual environment coping responses to flooding risks [5], improve the interpretability of disaster data and the effectiveness of decision making processes [6].

Virtual-Reality visualization of 3-D scenarios could allow users to view complex data in a more intuitive and comprehensible way and offers help in the communication of scientific knowledge.

This paper shows an example of the extension of the flood-mapping concept from an eminently cartographic context to a three-dimensional one, by applying two techniques for the representations of urban flood inundation in a virtual environment for hazard communication purposes.

The first procedure develops a simple and quick workflow based on the extrusion of building footprints from airborne LIDAR data and texture-mapping technique, to improve the realism of the building model façades. The results obtained with this approach allow one to provide an overall look at a neighbourhood level, but not at the level of the individual buildings in detail.

To reach this level, an accurate geometrical description of façade features is required, so the second technique required additional terrestrial laser scanning (TLS) acquisition, needed for 3-D city model reconstruction. This approach, more expensive and complex, could enhance the generation of a 3-D metric product with building details.

A comparative analysis of the two approaches for risk communication is presented, highlighting the level of detail, the pro and cons associated to the use of these procedures and analysing how the visualization outputs of the proposed procedures can be used in practice. These issues are discussed with reference to a potential flooding of the Crati River, in the old town of Cosenza (South of Italy). The hydraulic analysis of the flood evolution and the simulations details are not discussed here because they go beyond the purpose of the work and, in any case, can be found in [10].

\section{Case study description and traditional flood hazard map}

Cosenza is a town located at the confluence of two rivers, the Busento and the Crati. Around this confluence, the town was developed and the historical series of the floods that have affected the town over the centuries highlights a real risk of flooding in these areas. Figure 1 shows the simulated maximum water levels displayed according to the classic representation of a hazard map. The calculation refers to a hypothetical flood with a return time of 500 years. The hydraulic simulations have been carried out using a 2-D numerical model, previously developed and applied in several situations $[7,8,9]$, based on the shallow water equations. The details of the calculation on the Crati river can be found in [10,11]. Water depths are represented according to various shades of blue. Although the map shows the different water depths for inundated areas, inexperienced people may find it difficult to perceive the real hazard conditions, which characterize each point of the town. It is evident that from this representation it is not easy to realize the level at which water reaches the various points of the streets and the facades of buildings. These limitations can be overcome using a 3-D visualization.

The two techniques proposed in section 3 has been applied to the part of the domain that has been flooded by the Crati River over the last few centuries. 


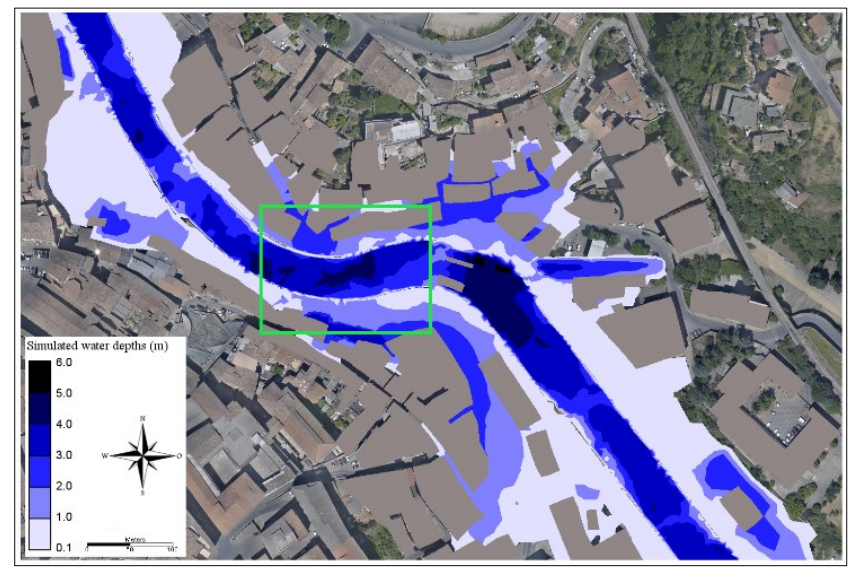

Figure 1. Maximum water levels represented by means of a classical flood hazard map. In green: the area considered in section 3 for the 3 -D representation of the flood map.

\section{Representations of urban flood map in 3-D virtual environments}

The following sections aim to perform an explorative analysis about the use of alternative techniques for 3-D representation of urban flood map. In particular, two techniques are proposed, compared and discussed.

\subsection{A quick and simple workflow based on texture mapping}

As far as the 3-D visualization of hydraulic results is concerned, the workflow is based on two main steps:

1. 3-D building reconstruction and modelling for realistic visualization purposes;

2. visual quality improvement of virtual urban environment and final renderings.

In particular, buildings extruded from their 2-D footprints in the shape file format in combination with airborne LIDAR point cloud. A representative height of the building is found by averaging the heights of the LIDAR points inside the footprint, using some geoprocessing tools. Thus, a horizontal plane approximates the building roof.

Since the 3-D virtual city model is intended for understanding the flood hazard in urban area, the texture mapping technique is used to improve the realism of the building model façade. For this, Google Maps Street View imagery was used for insert texture on the façades of buildings, giving them a realistic and recognisable look.

For improving the visualization quality, the buildings and the grids related to the ground level and the maximum water levels were imported in the open-source software Blender ${ }^{\mathrm{TM}}$. Then, materials and textures are created and assigned to the World environment, computational grid and water surface mesh. Materials, lights and backgrounds are welldefined using a shading nodes network. These nodes output shaders, colours, values and vectors.

More details about the workflow implementation are discussed in [12].

\subsubsection{Strong points and limitations}

Figure 2(a) represents an example of a three-dimensional representation of the information content in a traditional flood map. In particular, Figures 2(a) and 2(b), compared 
to the flood hazard map shown in figure 1, might add further information about the interaction between flood level and the public-private goods and better imagine how severe the flood is in that area.

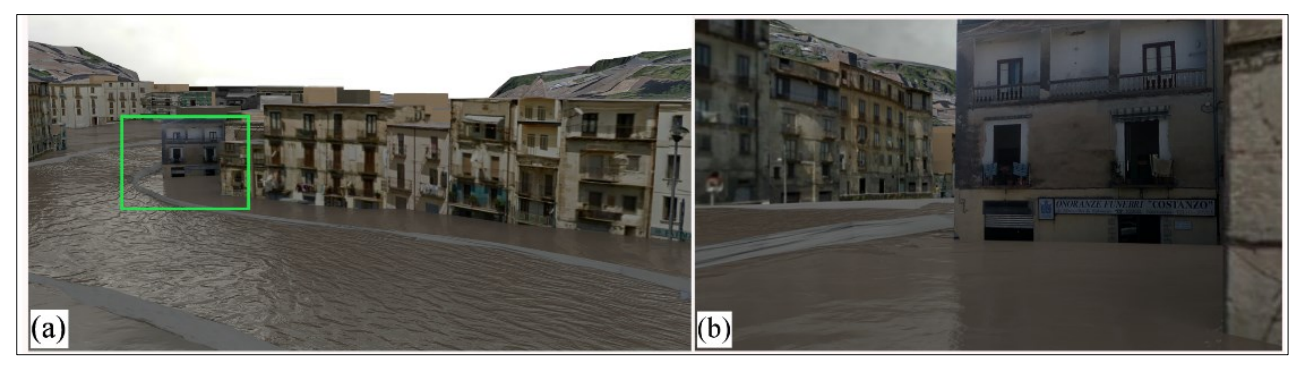

Figure 2. (a) An example of 3-D representation of the flood map using texture mapping; (b) visualization of the interaction between flood level and building façade highlighted in the green box (see Figure 2a).

For example, people living in those buildings can roughly realize the behaviour that they should have in case of flooding: to survive on the first floor or the roof, for example, or whether evacuation is necessary.

However, the figure 2 reveals some limits. In particular, building façades are characterized by planar polygons. This modelling approach is satisfactory for orthogonal views. For oblique views, however, the differences between the 3-D model and the real façade geometry reproduced in the photo textures are evident, especially in the presence of balconies, bows, corbels and so on. The absence of parallax points out the flatness of the surface. The photo textures quality is related to camera and standpoint; in narrow or jammed streets, there is the risk of taking photo of façade with vehicles, vegetation or pedestrians flattened on it.

The workflow presented in this section is relatively easy to implement in all the steps.

The results obtained with this approach allow one to provide an overall look at a neighbourhood level, but not at the level of the individual buildings in detail.

\subsection{Approach based on Terrestrial Laser Scanning acquisition}

To obtain a fully 3-D description of the environment of interest, laser-scanning technology has emerged in recent years as a leading source for automated acquisition of buildings, vegetation, terrain, etc., mainly due to the direct measurements of the surface topography both accurately and densely. In fact, 3D environment reconstruction with LiDAR data is taking on a central role in an increasing number of applications ranging from cultural heritage protection [13], urban planning, environmental impact assessment [14, 15] to disaster preparedness [16] and emergency management. Airborne data usually cover large urban areas, well recording structures on roofs and grounds. In contrast, terrestrial systems record information from building façades, but can hardly reach buildings' tops. Depending on the purpose of the product to be implemented, data from both sources can be integrated.

Compared to traditional survey techniques, terrestrial 3D laser scanners can provide faster, better quality, more precise analysis and objects reconstruction. Its use in highresolution urban flood studies would be beneficial in urban hydraulic models [17]. This approach, more expensive and complex, has enhanced the generation of 3-D metric products and surface modelling, capturing all topographic and building details to generate higher-level detailed model. In this context, particular emphasis should be placed on the issues related to the acquisition, management and processing of data acquired by TLS, for the construction of a geometrically valid and realistic virtual environment. 
A local survey of the building façades, streets and levees was made in the area of interest. Generally, the terrestrial laser scanning procedure required some steps, including: the project planning, scanning, registration/georeferencing, filtering and visualization.

Before the acquisition, strong emphasis has to be put on project planning in order to evaluate creating the geodetic network (if needed), checking field-of-view, and planning scan station locations. Data acquisition was performed using Leica ScanStation P20 laser scanner. In Table 1, some details about instrument and the way it has been used in the test field are reported.

\begin{tabular}{|l|l|}
\hline Type & $\begin{array}{l}\text { Ultra-high speed time-of-flight enhanced by Waveform } \\
\text { Digitising (WFD) technology }\end{array}$ \\
\hline 3D position accuracy & $6 \mathrm{~mm} @ 100 \mathrm{~m}$ \\
\hline Range & Up to $120 \mathrm{~m} ; 18 \%$ reflectivity (minimum range $0.4 \mathrm{~m}$ ) \\
\hline Field-of-View & Horizontal $360^{\circ}$, Vertical $270^{\circ}$; parallax-free \\
\hline Camera & Integrated $5 \mathrm{MP}$ digital camera, auto-adjusts to ambient lighting \\
\hline Weight & $11.9 \mathrm{~kg}$, nominal (w/o batteries) \\
\hline
\end{tabular}

Table 1. Summary of main technical specifications of Leica ScanStation P20 ; features refer to vendor documents.

Control points have been localized with a global navigation satellite system (GNSS) receiver. These targets have been used for georeferencing scans captured and for global point cloud reconstruction. The acquisition made it possible to obtain information on coordinates and colour of points - intensity and RGB values - with scanning resolution (distance between points) of $6 \mathrm{~mm}$ to $10 \mathrm{~m}$. The raw data from each scanning location was georeferenced, registered and filtered using the Leica Cyclone 9.0 (Leica Geosystems, AG) and the opensource software CloudCompare. Registration process makes it possible to merge several scans with each other in correct within a single coordinate system. Filtering process allows to automatically remove spurious signals that provide incorrect $3 \mathrm{D}$ coordinate.

\subsubsection{Preliminary visualization results}

An initial screening level on the usability of terrestrial LiDAR surveys for flood hazard communication purposes was conducted directly in the 3D point cloud processing software CloudCompare. The figure 3(a) shows the properties of point cloud scans, coupled to output of water depths in single band pseudo-colour. The user can determinate local building inundation directly by picking points on façade, in order to display corresponding local $\mathrm{z}$ coordinate (see the Figure 3(b) ). Furthermore, it should be observed that pictures like those shown here allow a better understanding of water levels situations around the buildings, highlighting that they can be variable throughout the building and denoting a not horizontal behaviour of water surfaces neat the buildings themselves.

3-D representation based on terrestrial LiDAR data may enable water experts to explore better flood protection mechanisms on large scales and promote the discussions between practitioners and decision makers in developing efficient worst-case-scenario and evacuation plans.

Water surface is not projected realistically into this $3 \mathrm{D}$ visualization, because the software used do not have a render engine. In addition, visualisation of point clouds is slow and nonintuitive, e.g., with sparseness of the points increasing with zoom.

This approach might be improved following two different options. The first one consists in a rendering system development as solution that combines large-scale LIDAR data and realistic water visualizations [18]. The second could be represented by the software 
framework use to extract precise models of urban environments from 3D point-cloud data [19].

The challenge of this approach is to provide rapid visualisation, manipulation and analysis of complex scenes, overcoming the gap between large-scale mapping LiDAR data and software and hardware systems (render engines and associated graphics cards).
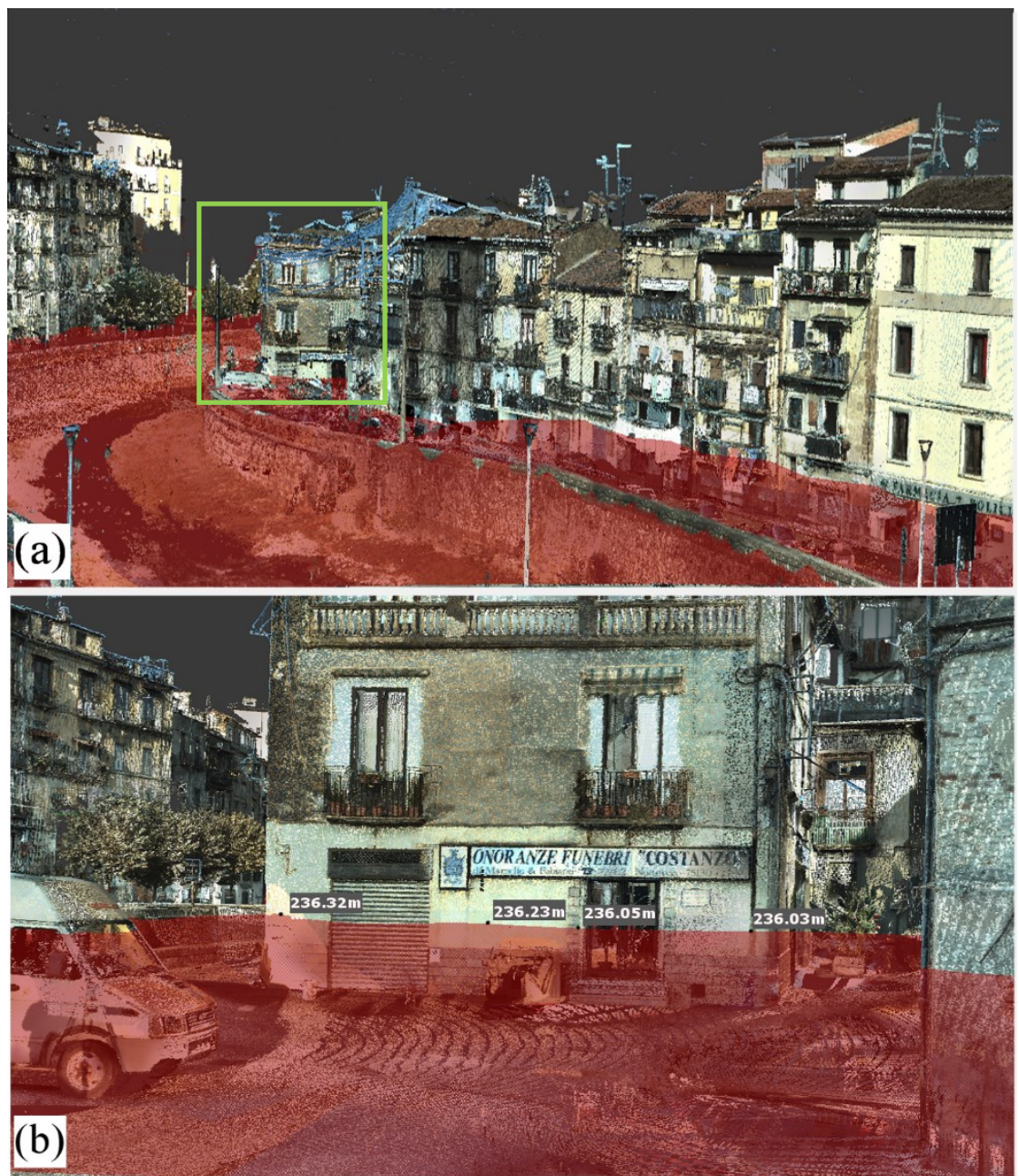

Figure 3. (a) An example of 3-D representation of the flood map using TLS data; (b) visualization of the interaction between flood level and building façade highlighted in the green box (see Figure 3a). Water surface depicted in red.

\section{Conclusions}

The traditional flood hazard maps, representing the flood-prone areas by chromatic shading or contour based resulting from 2-D hydraulic numerical modelling; do not allow a nonexpert audience an immediate readability of flooding effects. Consequently, these kind of representations could be not helpful to all the stakeholders involved in flood risk management and, therefore, different approaches have to be proposed in order to enhance flood hazard 
perception, to raise awareness on the related potential and to influence the behaviour of people in emergency situations.

Within this framework, the paper has suggested the extension of the flood-mapping concept from an eminently cartographic context to a three-dimensional one, by applying two techniques for the representations of urban flood inundation in a virtual environment. The first procedure proposed here is a simple and quick workflow based on the extrusion of building footprints from airborne LIDAR data and texture-mapping technique, to improve the realism of the building model façades. The second one required the additional terrestrial laser scanning acquisition, for the 3-D city model reconstruction.

The results depicted in the paper highlighted a significant level of realism for both the procedures. Representations like those shown in the paper might be very useful to improve the global awareness of the flood severity and the related potential damages. However, each procedure has own advantages and drawbacks.

In particular, the first procedure is quick and simple, does not require particular efforts in pre- and post-processing steps and allows getting more attractive scene, thanks to Blender ${ }^{\mathrm{TM}}$ render engine. Conversely, this approach can produce several distortions due to photo quality and stretching and pinching problems in the textured façades. This aspect must be considered carefully in quantitative evaluation about water level close to buildings. This information could not be fundamental if the intent is to provide a general indication to the population on the possible scenario associated with the flood but it could be essential for assessing the levels reached by water at single façade's scale.

The second procedure aims just to overcome these limits. Starting from point cloud data, in fact, it is possible not only to avoid deformations of the building but also to get hydraulic information about water depths around buildings. The very high point density may require considerable computational efforts; therefore, this procedure is preferable for detailed assessment on the extent of the flood levels in densely built-up urban areas.

The ideas discussed in this paper may be useful not only for academic researchers but also for both a professional audience and all the technicians working on environmental software development.

\section{References}

1. J. Pile, C. Gouramanis, A. D. Switzer, B. Rush, I. Reynolds, J. L. A. Soria. Int. J. Disaster Risk. Reduct. 27, 439-450. (2018).

2. J.G. Leskens, M. Brugnach, A.Y. Hoekstra, W. Schuurmans. Environ. Model. Softw. 53, 53-61. (2014).

3. X. Delgado-Galván, J. Izquierdo, J. Benítez, R. Pérez-García, Environ. Model. Softw. 51, 310-322. (2014).

4. S.A. Maskrey, N.J. Mount, C.R. Thorne, I. Dryden. Environ. Modell. Soft. 82, 275-294. (2016).

5. R. Zaalberg, C.J.H. Midden. Risk Anal. 33(5), 866-876. (2013).

6. L. Qiu, Z. Du, Q. Zhu, Y. Fan. Environ. Modell. Soft. 91, 111-126. (2017).

7. P. Costabile, C. Costanzo, F. Macchione. Appl. Numer. Math. 116, 141-156. (2017)

8. F. Macchione, P. Costabile, C. Costanzo, G. De Lorenzo, B. Razdar. J. Hydroinform. 18 (4), 615633. (2016).

9. C. Costanzo, F. Macchione. RiverFlow, 1, 291-301. (2006).

10. P. Costabile, F. Macchione. Environ. Modell. Soft. 67, 89-107. (2015).

11. P. Costabile, F. Macchione, L. Natale, G. Petaccia. CMES COMP MODEL ENG. 109(2). 81-103. (2015). 
12. F. Macchione, P. Costabile, C. Costanzo, R. De Santis. Submitted to Environ. Modell. Soft. (2018).

13. F. Remondino, A. Rizzi. Applied Geomatics, 2(3), 85-100. (2010).

14. K. Maragkogiannis, D. Kolokotsa, E. Maravelakis, A. Konstantaras. Sustain. Cities Soc. 13, $207-$ 216. (2014).

15. L. Wallace, V. Gupta, K Reinke, S. Jones. Remote Sens, 8(8). (2016).

16. G. Bui, P. Calyam, B. Morago, R. B. Antequera, T. Nguyen, Y. Duan. In 2015 IFIP/IEEE International Symposium on Integrated Network Management (IM). 790-793. (2015).

17. T. J. Fewtrell, A. Duncan, C. C. Sampson, J. C. Neal, P. D. Bates. Physics and Chemistry of the Earth, Parts A/B/C, 36(7), 281-291. (2011)

18. J.G Leskens, C. Kehl, T. Tutenel, T. Kol, G. de Haan, G. Stelling, E. Eisemann. Mitig. Adapt. Strat Gl. 22(2), 307-324. (2017).

19. B. Risse, M. Mangan, W. Stürzl, B. Webb. Environ. Model. Softw, 99, 88-100. (2018). 